\title{
TITLE:
}

\section{Histologic and Clinical Follow-up of Thyroid Fine Needle Aspirates in Pediatric Patients}

Kristen Partyka, M.D. '; Eric C. Huang, M.D., Ph.D. ${ }^{2}$; Harvey Cramer, M.D. ${ }^{1}$; Shaoxiong Chen, M.D., Ph.D. ; and Howard H. Wu, M.D. ${ }^{1}$

${ }^{1}$ Department of Pathology and Laboratory Medicine, Indiana University School of Medicine, Indianapolis, Indiana

${ }^{2}$ Department of Pathology and Laboratory Medicine, University of California, Davis Medical Center, Sacramento, California

RUNNING TITLE: Pediatric thyroid FNA

Corresponding author:

Howard H. Wu, MD

Indiana University School of Medicine

IU Health Pathology Laboratory

$350 \mathrm{~W} 11^{\text {th }} \mathrm{St}$, IUHPL \#4086

Indianapolis, IN 46202

Telephone: $\quad 317-491-6154$

Facsimile: 317-491-6419

Email: hhwu@iupui.edu

Total number of text pages: 10; tables: 3; figures: 1

Disclosures: There are no prior publications or submissions with any overlapping information, including studies and patients. This manuscript has not been and will not be submitted to any other journal while it is under consideration. Each author listed on the manuscript has seen and approved the submission of this version of the manuscript and takes full responsibility for the manuscript. The authors have no financial or conflicts of interest to disclose. This research received no specific grant from any funding agency in the public, commercial, or not-for-profit sectors.

Precis: In our experience, the risk of malignancy of thyroid FNA for the pediatric population $(\leq 18$ years old), not including papillary microcarcinoma, was $2 \%$ for benign aspirates, $21 \%$ for AUS, $57 \%$ for FN, and $100 \%$ for suspicious or malignant aspirates.

This is the author's manuscript of the article published in final edited form as: Partyka, K. L., Huang, E. C., Cramer, H. M., Chen, S., \& Wu, H. H. (2016). Histologic and clinical follow-up of thyroid fine-needle aspirates in pediatric patients. Cancer Cytopathology, 124(7), 467-471. http://doi.org/10.1002/cncy.21713 


\begin{abstract}
:
BACKGROUND: Although fine needle aspiration (FNA) has an important role in evaluating thyroid
\end{abstract} nodules in adults, there is little published data regarding its utility in the pediatric population.

METHODS: A retrospective analysis of thyroid FNAs on patients $\leq 18$ years of age at two institutions was conducted. Aspirates were retrospectively categorized using the Bethesda System for Reporting Thyroid Cytopathology (TBSRTC). These diagnoses were then correlated with either final histopathology or clinical follow-up.

RESULTS: A total of 186 thyroid FNAs from 154 patients (122 females, 32 males), ranging from 9 months to 18 years (median 16 years, mean 14 years), were identified. FNA was performed to evaluate one to three nodules for each patient. Aspirates were classified as follows: non-diagnostic (n=27), benign $(n=114)$, atypia of undetermined significance (AUS, $n=21)$, follicular neoplasm $(F N, n=8)$, suspicious for malignancy $(n=3)$, and malignant $(n=13) .61$ samples had histologic correlation, 68 were followed clinically for $\geq 2$ years, and 57 had either no follow-up or were followed for $<2$ years. For statistical purposes, FNA diagnoses of suspicious and malignant were considered positive, and benign lesions were negative. The accuracy was $99 \%$, and the sensitivity and specificity were $94 \%$ and $100 \%$, respectively. The risk of malignancy, not including papillary microcarcinoma, was $2 \%$ for benign aspirates, $21 \%$ for AUS, $57 \%$ for FN, and $100 \%$ for suspicious or malignant aspirates.

CONCLUSIONS: This analysis demonstrates that FNA is a sensitive and highly specific modality for evaluating thyroid nodules in pediatric patients. Each diagnostic category can facilitate communication and guide appropriate management.

KEYWORDS: thyroid, FNA, cytology, the Bethesda System, pediatric 


\section{TEXT:}

\section{Background:}

Thyroid nodules are relatively uncommon in the pediatric population, with an estimated prevalence ranging from $1-2 \% .^{1,2}$ However, the rate of malignancy in these nodules is higher in children compared to adults. ${ }^{3-7}$ The average incidence of thyroid carcinoma in patients with thyroid nodules ranges from $9.2-50 \%$ in various studies with an overall reported risk of $26.4 \%{ }^{8}$ This data can lead to a more aggressive approach to the clinical management of thyroid nodules in pediatric patients. Total thyroidectomies and diagnostic lobectomies are associated with an inherent risk of complications. Thyroid fine needle aspiration (FNA) has gained widespread acceptance as a useful modality for selecting appropriate patients for surgery while also identifying those who can be safely monitored by clinical follow-up. FNA has an important and proven role in evaluating thyroid nodules in adults, but it has been used less extensively in children. There is very little published data regarding its diagnostic utility in the pediatric population. Despite some concerns regarding its limitations, FNA is a safe and effective method, and it can be utilized to guide clinical decisions. ${ }^{9}$ FNA should play a central role in the assessment and management of pediatric patients who present with thyroid nodules.

\section{MATERIALS AND METHODS:}

The study was approved by the Institutional Review Boards of Indiana University and University of California, Davis (UC Davis). A retrospective analysis of thyroid FNAs performed at Indiana University Health (IU Health) and UC Davis Medical Center on patients $\leq 18$ years of age was conducted. It spanned a 20-year period from 1995-2014 at IU Health and a 10-year period from 20052014 at UC Davis Medical Center. All aspirates were retrospectively categorized into six groups using the Bethesda System for Reporting Thyroid Cytopathology (TBSRTC). ${ }^{10}$ A computer search of the anatomic pathology archives and the electronic medical record was performed to document the final histopathology of patients who underwent surgery. Also, the electronic medical record was utilized to track those patients who were monitored with close clinical follow-up alone. Clinical follow-up 
consisted of neck ultrasounds and clinic notes with documented histories and physical examinations. The cytologic diagnoses were then correlated either with the pathology reports or with the clinical follow-up.

\section{RESULTS:}

A total of 186 thyroid FNA samples from 154 patients (122 females, 32 males) with an age range of 9 months to 18 years (median 16 years, mean 14 years) were identified at IU Health and UC Davis Medical Center. FNAs were performed to evaluate one to three nodules for each patient. Among these subjects, 127 patients had 1 nodule with 1 FNA performed. 10 patients had 1 nodule with 1 initial FNA and 1 repeat FNA. 12 patients had 2 nodules with a single FNA performed on each nodule. Also, 1 patient had 2 nodules with an initial FNA on each nodule and 1 repeat FNA on one nodule. Lastly, 4 patients had 3 nodules with a separate FNA on each nodule. Among 11 patients with repeat FNA, the results showed 3 patients with a benign diagnosis in both initial and repeat aspirates. 7 patients were non-diagnostic on initial aspirates and benign on follow-up, and 1 patient showed benign on initial aspirate and AUS on repeat FNA. Surgical follow-up revealed a benign nodule in this patient who was diagnosed AUS on repeat FNA.

The aspirates were classified according to TBSRTC as follows: non-diagnostic $(n=27,14.5 \%)$, benign $(n=114,61.3 \%)$, atypia of undetermined significance (AUS, $n=21,11.3 \%)$, follicular neoplasm (FN, $n=8,4.3 \%)$, suspicious for malignancy $(n=3,1.6 \%)$, and malignant $(n=13,7.0 \%)$. Overall, $61(32.8 \%)$ FNA samples had surgical follow-up with histologic correlation, and 68 (36.6\%) FNAs were followed clinically for $\geq 2$ years. $57(30.6 \%)$ FNAs had either no follow-up or were followed for $<2$ years (Table $1)$.

For statistical purposes, FNA diagnoses of suspicious and malignant were categorized as positive, and all benign lesions were designated as negative. Clinical follow-up for $\geq 2$ years with no documented 
change in physical exam or neck ultrasound was considered a benign result. Also, those patients with no follow-up, follow-up for $<2$ years, or increased nodule size on ultrasound were not included in these calculations. Among the cases with surgical correlation, the pathology reports showed benign thyroid disease, follicular adenoma, papillary microcarcinoma, and malignancy (Table 2).

The overall accuracy of thyroid FNA in this population was found to be $99 \%$, and the overall sensitivity and specificity were $94 \%$ and $100 \%$, respectively. The risk of neoplasm was $9 \%$ for benign aspirates, $53 \%$ for AUS, $100 \%$ for FN, and $100 \%$ for suspicious or malignant aspirates (Figure 1). The risk of malignancy, not including papillary microcarcinoma, was $2 \%$ for benign aspirates, $21 \%$ for AUS, $57 \%$ for FN, and $100 \%$ for suspicious or malignant aspirates (Figure 1). The risk of malignancy including papillary microcarcinoma was $2 \%$ for benign aspirates, $26 \%$ for AUS, $57 \%$ for FN, and $100 \%$ for suspicious or malignant aspirates.

Among the 25 surgical results of malignancy, there were 18 cases $(72 \%)$ of papillary thyroid carcinoma and 4 cases $(16 \%)$ of follicular variant of papillary thyroid carcinoma. Two cases $(8 \%)$ showed follicular carcinoma, and one case (4\%) demonstrated metastatic germ cell tumor (Table 3).

\section{DISCUSSION:}

This retrospective analysis demonstrates that FNA is a sensitive and highly specific diagnostic modality for evaluating thyroid nodules found in pediatric patients. There is little data published on the use of FNA in children, and the available reports comprise only small patient cohorts. In our analysis, the sensitivity of thyroid FNA was $94 \%$, and this is in agreement with previously published data showing a sensitivity ranging from $60-100 \%$. Also, the specificity was $100 \%$, which is concordant with reports of specificity ranging from $90-95 \% .{ }^{11-14}$ There is interest in refining the diagnostic approach to pediatric thyroid nodules, and this analysis lends support to that growing literature. 
The overwhelming majority of our patients with a benign diagnosis by FNA were monitored with clinical follow-up alone. There was a $2 \%$ risk of malignancy and a $9 \%$ risk of neoplasm among those patients with benign FNA diagnoses who subsequently underwent surgery or clinical follow-up for more than two years. With no documented changes in physical exam and/or neck ultrasound, there is some credence to monitoring these patients with clinical follow-up alone. On the opposite end of the spectrum, all of the patients with a suspicious or malignant aspirate had surgery. There was a $100 \%$ risk of neoplasm and a 100\% risk of malignancy in this subset of patients.

Twenty-seven patients (14.5\%) had a non-diagnostic aspirate in our study. Seven of these patients had repeat FNA and showed benign in the follow-up aspirates. A meta-analysis found $1.8 \%$ to $23.6 \%$ of all thyroid FNAs were non-diagnostic with an overall value of $12.9 \% .{ }^{15}$ It seems that our non-diagnostic rate is slightly higher, and this may be associated with the focus on the pediatric population.

The indeterminate categories of AUS and FN can often lead to uncertainties in clinical management. This retrospective analysis illustrates a 21\% (excluding papillary microcarcinoma) and 26\% (including papillary microcarcinoma) risk of malignancy in the group of AUS aspirates and a $57 \%$ risk of malignancy in the group of FN aspirates. A meta-analysis ${ }^{15}$ showed FNA cases in the AUS category ranged from $3 \%$ to $27.2 \%$ with an overall value of $9.6 \%$ and an overall rate of malignancy of $15.9 \%$. The FNA cases in the FN category ranged from $1.2 \%$ to $25.3 \%$ with an overall value of $10.1 \%$ and a risk of malignancy of $26.1 \%$. There is an incremental risk of malignancy for the pediatric population within these categories, and this is consistent with most other reports in the literature. ${ }^{6,16-18}$ FNA can be an effective means for clinicians to risk stratify thyroid nodules. ${ }^{6}$ Overall, the accuracy of the thyroid FNA was $99 \%$ in this analysis, which is slightly superior compared to previous reports of $70-95 \%$ accuracy. ${ }^{2,4,12}$ 
The clinical management of thyroid nodules varies across institutions, and FNA is often underutilized in the pediatric population. Since the risk of malignancy is high in these lesions, a systematic approach to the management of these patients is warranted. ${ }^{3}$ Total thyroidectomies and diagnostic lobectomies are established components of the multidisciplinary management of children who present with thyroid nodules. However, these surgical procedures carry a risk for perioperative complications, including hypoparathyroidism and recurrent laryngeal nerve injury., ${ }^{419}$ In patients younger than 10 years of age, the risk of complications increases with repeat surgery. ${ }^{16}$ Thyroid FNA has the potential to decrease the number of patients undergoing unnecessary operations while simultaneously increasing the percentage of patients with malignancy who are referred for surgery. ${ }^{20,21}$

FNA is a highly accurate, minimally invasive procedure that can add valuable information for clinical management of patients with thyroid nodules. This diagnostic technique can be safely and easily used in children with minimal discomfort. ${ }^{5,12}$ It is well tolerated and can be performed with ultrasound guidance, local anesthetics, and pediatric distraction techniques. ${ }^{5}$ This study shows that FNA is a sensitive and highly specific tool for the evaluation of thyroid lesions occurring in children. If a nodule is found to be malignant on FNA, the patient can be referred for definitive surgery, and the need for an intraoperative frozen section is usually obviated. When the cytology is benign, our results demonstrate a low risk of malignancy, and it is therefore reasonable to manage these patients with clinical follow-up alone. Additionally, if the FNA is indeterminate, our analysis shows a distinct incremental risk of malignancy in these categories. Although not performed in our series, molecular testing was proven to have value in management of these indeterminate thyroid aspirates in pediatric patients. ${ }^{17}$ Detection of a molecular genetic abnormality is correlated with a $100 \%$ malignancy rate and would enable these patients to go directly to thyroidectomy without a need for repeat FNA. The FNA diagnosis should be correlated with nodule size, clinical symptoms, and radiologic findings. ${ }^{6}$ Each diagnostic category can 
hopefully facilitate communication with physicians and guide appropriate clinical management in these pediatric patients.

\section{References}

1. Raab SS, Silverman JF, Elsheikh TM, Thomas PA, Wakely PE. Pediatric Thyroid Nodules: Disease Demographics and Clinical Management as Determined by Fine Needle Aspiration Biopsy. Pediatrics. 1995;95: 46-49.

2. Stevens C, Lee JKP, Sadatsafavi M, Blair GK. Pediatric thyroid fine-needle aspiration cytology: a meta-analysis. J Pediatr Surg. 2009;44: 2184-2191.

3. The Canadian Pediatric Thyroid Nodule (CaPTN) Study Group. The Canadian Pediatric Thyroid Nodule Study: an evaluation of current management practices. J Pediatr Surg. 2008;43: 826-830.

4. Khozeimeh N, Gingalweksi C. Thyroid Nodules in Children: A Single Institution's Experience. J Oncol. 2011;2011: 974125.

5. Gupta A, Ly S, Castroneves LA, et al. A Standardized Assessment of Thyroid Nodules in Children Confirms Higher Cancer Prevalence Than in Adults. J Clin Endocrinol Metab. 2013;98(8): 32383245 .

6. Smith M, Pantanowitz L, Khalbuss WE, Benkovich VA, Monaco SE. Indeterminate Pediatric Thyroid Fine Needle Aspirations: A Study of 68 Cases. Acta Cytol. 2013;57(4): 341-348. 
7. Lale SA, Morgenstern NN, Chiara S, Wasserman P. Fine Needle Aspiration of Thyroid Nodules in the Pediatric Population: A 12-Year Cyto-Histological Correlation Experience at North Shore-Long Island Jewish Health System. Diagn Cytopathol. 2015;43(8): 598-604.

8. Niedziela M. Pathogenesis, diagnosis and management of thyroid nodules in children. Endocr Relat Cancer. 2006;13: 427-453.

9. Bargren AE, Meyer-Rochow GY, Sywalk MS, Delbridge LW, Chen H, Sidhu SB. Diagnostic Utility of Fine-Needle Aspiration Cytology in Pediatric Differentiated Thyroid Cancer. World J Surg. 2010;34: 1254-1260.

10. Cibas ES, Ali SZ. The Bethesda System for Reporting Thyroid Cytopathology. Am J Clin Pathol. 2009;132: 658-665.

11. Lugo-Vicente H, Ortiz VN, Irizarry H, Camps JI, Pagan V. Pediatric Thyroid Nodules: Management in the Era of Fine Needle Aspiration. J Pediatr Surg. 1998;33: 1302-1305.

12. Arda IS, Yildirim S, Demirham B, Firat S. Fine needle aspiration biopsy of thyroid nodules. Arch Dis Child. 2001;85: 313-317.

13. Amrikachi M, Ponder TB, Wheeler TM, Smith D, Ramzy I. Thyroid Fine-Needle Aspiration Biopsy in Children and Adolescents: Experience with 218 Aspirates. Diagn Cytopathol. 2005;32: 189-192.

14. Hosler GA, Clark I, Zakowski MF, Westra WH, Ali SZ. Cytopathologic Analysis of Thyroid Lesions in the Pediatric Population. Diagn Cytopathol. 2006;34: 101-105. 
15. Bongiovanni M, Spitale A, Faquin WC, Mazzucchelli L, Baloch ZW. The Bethesda System for Reporting Thyroid Cytopathology: A Meta-Analysis. Acta Cytol. 2012;56: 333-339.

16. Norlén O, Charlton A, Sarkis LM, et al. Risk of malignancy for each Bethesda class in pediatric thyroid nodules. J Pediatr Surg. 2015;50: 1147-1149.

17. Monaco SE, Pantanowitz L, Khalbuss WE, et al. Cytomorphological and Molecular Genetic Findings in Pediatric Thyroid Fine-Needle Aspiration. Cancer Cytopathol. 2012;120: 342-50.

18. Rossi ED, Straccia P, Martini M, et al. The Role of Thyroid Fine-Needle Aspiration Cytology in the Pediatric Population. Cancer Cytopathol. 2014;122: 359-67.

19. Francis, GL. Management Guidelines for Children with Thyroid Nodules and Differentiated Thyroid Cancer: The American Thyroid Association Guidelines Task Force on Pediatric Thyroid Cancer. Thyroid. 2015;25(7): 716-759.

20. Hamberger B, Gharib H, Melton LJ III, Goellner JR, Zinsmeister AR. Fine-Needle Aspiration Biopsy of Thyroid Nodules: Impact on Thyroid Practice and Cost of Care. Am J Med. 1982;73: 381384.

21. Gharib H, Zimmerman D, Goellner JR, Bridley SM, LeBlanc SM. Fine-Needle Aspiration Biopsy: Use in Diagnosis and Management of Pediatric Thyroid Diseases. Endocr Pract. 1995;1(1): 9-13. 


\section{FIGURE LEGEND:}

Figure 1 Risk of Neoplasm and Malignancy associated with each Diagnostic Category 